\title{
Concurrent Complementary and Alternative Medicine CAM and Conventional Rehabilitation Therapy in the Management of Children with Developmental Disorders
}

\author{
Soo Yeon Kim, ${ }^{1,2}$ Yong-Il Shin, ${ }^{1,2}$ Sang-Ook Nam, ${ }^{2,3}$ Chang-Hyung Lee, ${ }^{1,2}$ Yong Beom Shin, ${ }^{4}$ \\ Hyun-Yoon Ko, ${ }^{1,2}$ and Young-Ju Yun ${ }^{5}$ \\ ${ }^{1}$ Department of Rehabilitation Medicine, Pusan National University Yangsan Hospital, Pusan National University School of Medicine, \\ Yangsan, Republic of Korea \\ ${ }^{2}$ Research Institute for Convergence of Biomedical Science and Technology, Pusan National University Yangsan Hospital, \\ Yangsan, Republic of Korea \\ ${ }^{3}$ Department of Pediatrics, Pusan National University Yangsan Hospital, Pusan National University School of Medicine, \\ Yangsan, Republic of Korea \\ ${ }^{4}$ Department of Rehabilitation Medicine, Pusan National University Hospital, Pusan National University, School of Medicine, \\ Busan, Republic of Korea \\ ${ }^{5}$ Department of Integrative Medicine, School of Korean Medicine, Pusan National University Yangsan Hospital, \\ Yangsan, Republic of Korea \\ Correspondence should be addressed to Young-Ju Yun; mdkmdyun@pusan.ac.kr
}

Received 12 July 2013; Revised 22 September 2013; Accepted 23 September 2013

Academic Editor: Gloria Brusotti

Copyright (C) 2013 Soo Yeon Kim et al. This is an open access article distributed under the Creative Commons Attribution License, which permits unrestricted use, distribution, and reproduction in any medium, provided the original work is properly cited.

Background. We investigated the concurrent use of conventional rehabilitations and complementary and alternative medicine (CAM) therapies for the long-term management of children with developmental disorders (DDs). Methods. The parents or caregivers of 533 children with DDs (age range, 1-19 years) who visited the rehabilitation centers were surveyed using in depth face-to-face interviews. Results. Of the 533 patients enrolled, 520 completed the questionnaire (97\% response rate). A total of $292(56 \%)$ children were receiving multiple therapies, more than two conventional rehabilitations and CAM, at the time of the interview. A total of 249 (48\%) children reported lifetime CAM use, 23\% used CAM at the time of the interview, and $62 \%$ of the patients planned to use CAM therapy in the future. Conventional rehabilitation therapies used at the time of the interview included physical therapy (30\%), speech therapy (28\%), and occupational therapy (19\%), and the CAM therapies included herbal medicine (5\%) and acupuncture or moxibustion (3\%). The respondents indicated that in the future they planned to use acupuncture or moxibustion (57\%), occupational therapy (18\%), cognitive behavioral therapy (16\%), speech therapy (10\%), and physical therapy (8\%). Conclusion. Concurrent management as conventional rehabilitations and CAM therapies is widely used by children with DDs.

\section{Introduction}

Children with developmental disorders (DDs) do not achieve developmental milestones in motor, perceptual, speech, cognition, and behavioral areas. DDs are relatively common with an estimated prevalence of 3 4\% worldwide [1]. Children with DDs are at risk of chronic physical, developmental, behavioral, and emotional conditions that need special treatment in addition to that required by healthy children. Early intervention has been shown to improve developmental outcomes and reduce the socioeconomic impact of DDs [1]; thus, children with DDs need a wide range of treatments.

Children with DDs often receive a combination of longterm conventional rehabilitation therapies, such as physical therapy, occupational therapy, speech therapy, counseling, and cognitive behavioral therapy, in multiple rehabilitation centers. The use of complementary and alternative medicine (CAM) to treat children is on the rise in developed countries 
TABLE 1: Characteristics of children with developmental disorder.

\begin{tabular}{lc}
\hline Characteristics & \\
\hline Age (year), mean \pm standard deviation & $7.5 \pm 2.3$ \\
Sex, $n(\%)$ & $296(57 \%)$ \\
$\quad$ Male & $224(43 \%)$ \\
$\quad$ Female & \\
Diagnosis, $n(\%)$ & $368(71 \%)$ \\
$\quad$ Epilepsy & $70(13 \%)$ \\
$\quad$ Cerebral palsy & $33(6 \%)$ \\
$\quad$ ADHD & $52(10 \%)$ \\
$\quad$ Others & $5 \pm 1.9$ \\
Disease durations (year), mean \pm & \\
standard deviation
\end{tabular}

ADHD: attention deficit hyperactivity disorder.

with a prevalence ranging from $1.8 \%$ to $80 \%$, depending on the population and study design [2-6]. A recent study reported that $36 \%$ of general pediatric patients and $61.9 \%$ of children with epilepsy used CAM in USA [7]. CAM includes homeopathy, phytotherapy, acupuncture, moxibustion, anthroposophic medicine, and Bach flower remedies.

Oriental medicine is considered a type of CAM by Western medicine practitioners [8]. CAM is an important form of healthcare in Korea and is an alternative treatment option for patients with DDs. Few studies have examined the concurrent use of conventional rehabilitation programs provided in multiple clinical settings and CAM to manage DDs. In acknowledgment of the increasing public interest in CAM, we assessed the motivation, prevalence of use (if ever used after the diagnosis of a DD), and perceived effectiveness of long-term conventional multicenter and CAM therapies in Korean patients with DDs.

\section{Materials and Methods}

Between March and August 2011, 533 children with DDs (age range, 1-19 years) from two hospitals in Korea were enrolled in the present study. We conducted face-to-face interviews with 533 parents or caregivers of the children about the use of the conventional rehabilitation therapies in multiple centers and CAM. The questionnaire was designed using previous data [3, 9-13], list of CAM and conventional rehabilitation therapies, and input from practitioners and was reviewed by a panel of experts. One researcher conducted all interviews taking care not to express personal views about conventional rehabilitation and CAM therapies. In addition, each patient underwent a $15 \mathrm{~min}$ questionnairebased interview conducted by a trained nurse in the hospitals' outpatient clinics. The questionnaire addressed four areas: children's characteristics (age, sex, diagnosis, and disease duration), concurrent management at the time of the survey, those in the past, and future plans for therapy. Concurrent management was defined as concurrent CAM and conventional rehabilitation therapy. Statistical tests were conducted using the Statistical Package for Social Sciences version 15
(SPSS Inc., Chicago, IL, USA), frequency analysis, and chisquare test. The study was approved by the local ethics committee.

\section{Results}

3.1. Demographic Characteristics. Of the 533 patients enrolled, 520 completed the questionnaire (97\% response rate). Of those, 296 were males and 224 were females. Mean age was $7.5 \pm 2.3$ years. The diagnoses were epilepsy, 368 (71\%), cerebral palsy, 70 (13\%), attention deficit hyperactivity disorder, $33(6 \%)$, and other such as mood disorders or musculoskeletal problems, 49 (10\%). The demographic characteristics of the survey respondents are summarized in Table 1.

3.2. Management Strategies. A total of 292 (56\%) children were receiving concurrent management at the time of the interview, and $25 \%$ had previously undergone concurrent management, but they were not receiving it at the time of the interview. Concurrent management used at the time of the interview was physical therapy (30\%), speech therapy (28\%), occupational therapy (19\%), counseling or cognitive behavioral therapy (8\%), herbal medicine (5\%), acupuncture or moxibustion (3\%), mineral and vitamin therapy $(13 \%)$, and manual therapies $(0.4 \%)$.

Treatments used more than once in the past were physical therapy (8\%), occupational therapy (9\%), speech therapy (8\%), counseling or cognitive behavioral therapy (3\%), herbal medicine $(13 \%)$, acupuncture or moxibustion (11\%), mineral and vitamin therapy (6\%), and manual therapies (3\%). Multiple responses were permitted for each question.

Finally, we assessed the need for continued therapy in the future. The results showed that $62 \%$ of the parents or caregivers were considering future treatment such as acupuncture or moxibustion (57\%), herbal medicine $(7 \%)$, mineral and vitamin therapy $(6 \%)$, manual therapies $(6 \%)$, occupational therapy (18\%), cognitive behavioral therapy (16\%), speech therapy (10\%), and physical therapy (8\%) (Figure 1).

There were no significant differences of concurrent management between boys and girls. Seventy-three percent of concurrent management users at present plan to use CAM in the future. There were significant differences for planning $\mathrm{CAM}$ in the future between concurrent management users and nonconcurrent management users statistically $(P<$ 0.05).

\subsection{Reasons for Discontinuing CAM or Conventional Reha-} bilitation Medicine. We found that $34 \%$ of the children had used CAM, and 23\% were using CAM at the time of the interview. Moreover, $26 \%$ of the children had used conventional rehabilitation therapy in the past, and $47 \%$ were using conventional rehabilitation therapy at the time of the interview. The reasons given for discontinuing CAM were improved condition, 39\%; noneffectiveness, 36\%; patient did not cooperate, $14 \%$; financial burden, $13 \%$; inconvenience (distance or time), 13\%; and side effects, 9\%. The reasons given for discontinuing conventional rehabilitation therapy 


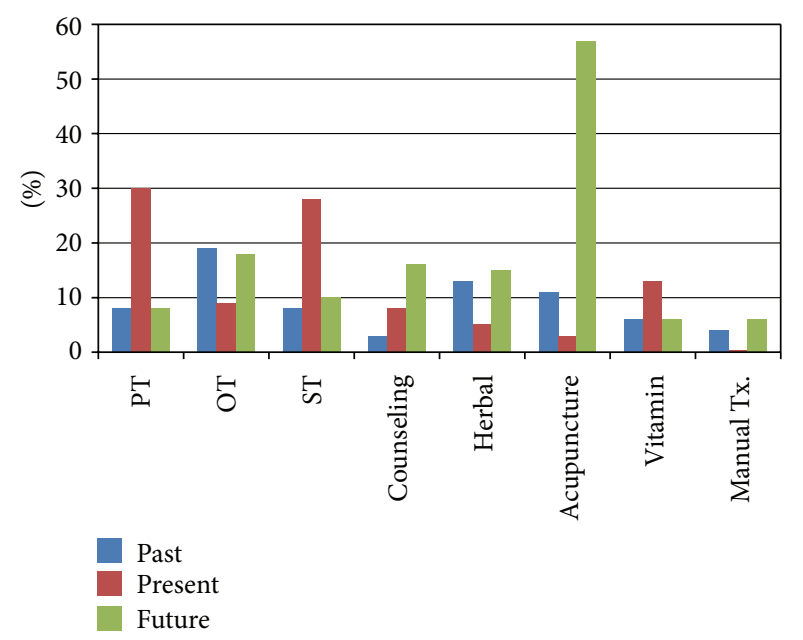

Figure 1: Types of rehabilitation treatment used by children with developmental disorders. Of 520 children with developmental disorders, 292 (56\%) used multiple conventional rehabilitation and CAM therapies administered in multiple centers. PT, physical therapy; OT, occupational therapy; ST, speech therapy.

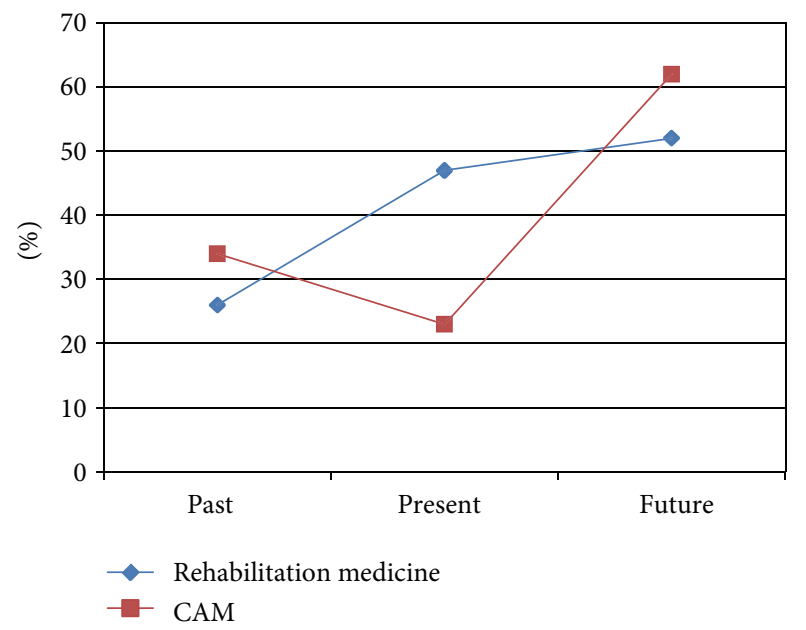

FIGURE 2: Trends in concurrent management for children with developmental disorders. Of 520 children with development disorders, 249 (48\%) reported lifetime CAM use, and 23\% were using $\mathrm{CAM}$ at the time of the interview. Furthermore, $62 \%$ of the patients planned to use CAM management in the future.

included inconvenience (distance or time), 33\%; noneffectiveness, $17 \%$; improved condition, $11 \%$; financial burden, $11 \%$; patient did not cooperate, $10 \%$; and side effects, $2 \%$.

\section{Discussion}

Various conditions can cause developmental delays, and children with DDs exhibit several clinical symptoms that may require long-term management; thus, a range of therapeutic options are necessary for these patients. Because of its chronic nature, physical medicine and rehabilitation are the cornerstones of DD treatment. Generally, children with
DDs undergo more than one type of therapy in multiple rehabilitation centers. However, a significant proportion of patients use, and frequently perceive a benefit from, alternative medicine [14], and CAM therapies are widely used by pediatric patients with DDs in Korea. The reasons parents and caregivers gave for choosing CAM therapies included positive views of the complementary therapies, limitations of conventional rehabilitation medicines, concern about the adverse effects of conventional medicine, doctors' recommendation, and the increased availability of complementary therapies [15-17]. In Korea, Traditional Oriental Medicine is performed by certified Korean oriental medical doctors; thus, patients tend to view CAM as more acceptable and natural than conventional medicine. The recent growing interest in CAM has stimulated publications on CAM treatment for various medical conditions [17]. In response to the increasing public interest in CAM, our survey assessed the expectations and prevalence of CAM among patients with DDs and their parents or caregivers.

Current estimates based on previous studies indicate that $19 \%$ to $57 \%$ of patients with DDs use CAM [3, 9-11]. In Korea, $54 \%$ of stroke patients [12] and $82 \%$ of patients with rheumatoid arthritis [13] have been reported to use CAM. In fact, the rate of CAM use is higher in people with poor health, chronic conditions, and/or a low functional status than in healthy people [18].

The present study found that 292 (56\%) children with DDs used concurrent conventional rehabilitation and CAM therapies administered in multiple settings. For example, patients may have enrolled in similar physical therapy programs in multiple rehabilitation centers, possibly because the parents/caregivers believed that the various rehabilitation facilities had different strong points. However, in using multiple centers, the patients risk high costs and inconsistent care across centers.

A total of 249 (48\%) of the children reported lifetime CAM use, $23 \%$ were using CAM at the time of the interview, and $62 \%$ of the patients planned to use CAM treatments in the future (Figure 2). Of the various CAM therapies, 57\% of patients planned to use acupuncture and moxibustion for future management. More higher percent of patients in concurrent management users plan to use CAM in the future compared to nonconcurrent management users. Our study showed wide use of, and an increasing demand for, CAM therapies.

The most common reasons for discontinuing CAM treatment were improved condition, noneffectiveness, and side effects, whereas inconvenience was the most common reason why patients discontinued conventional rehabilitation therapies.

The appropriate use of CAM involves engaging a medical doctor and oriental medical doctor and ensuring good communication between both doctors and the patient. Medical doctors must recognize that complementary medicines are widely used by patients with DDs and be aware of potential adverse effects. Physicians should determine which CAM therapies their patients use and advise them and their families about the evidence-based efficacy and side effects of the treatments. Intervention by the physician may be necessary 
because concurrent CAM and conventional rehabilitation therapies can inadvertently cause side effects or render the treatment ineffective. The wide range of CAM treatments used by the families of children with DDs in our study highlights the ongoing search for effective and balanced treatment for these lifelong conditions. The pediatric medical community needs to be aware of CAM use to provide better education, understanding, and optimal patient-physician relationships.

The present study is the first to investigate the use of concurrent conventional rehabilitation medicine and CAM for the management of children with DDs in Korea. We identified the types of CAM used by patients with DDs, those most commonly used, and reasons for discontinuing CAM.

Furthermore, we investigated the management of DDs using concurrent conventional rehabilitation therapies with overlapping programs in multiple centers and CAM.

The limitations of this study are cross-sectional design and being unable to make any conclusions about causality. This study reveals high percentage of caregivers who plan to use CAM in the future. Although one researcher conducted the interviews and did not express personal views about CAM or conventional rehabilitation therapies, just asking caregivers about these therapies could influence answers given. Participants were enrolled from only two hospitals in Korea; thus, the findings are not generalizable to all Korean children with DDs and limited to other children with DDs in other countries. However, our findings reveal trends in therapy preferences for children with DDs in Korea.

\section{Conclusion}

The present survey showed that a higher percentage of Korean children with DDs currently use multiple conventional rehabilitation therapies administered in overlapping programs; however, we found increasing interest in using CAM for future treatment. Our findings suggest that medical practitioners need to be aware of the various types of conventional and CAM therapies to provide comprehensive, integrated medical services for children with DDs.

\section{Acknowledgment}

This study was supported by Biomedical Research Institute Grant (2011-02), Pusan National University Hospital.

\section{References}

[1] M. C. Petersen, D. A. Kube, and F. B. Palmer, "Classification of developmental delays," Seminars in Pediatric Neurology, vol. 5, no. 1, pp. 2-14, 1998.

[2] M. P. Davis and P. M. Darden, "Use of complementary and alternative medicine by children in the United States," Archives of Pediatrics and Adolescent Medicine, vol. 157, no. 4, pp. 393396, 2003.

[3] W. L. Oppenheim, "Complementary and alternative methods in cerebral palsy," Developmental Medicine and Child Neurology, vol. 51, no. 4, pp. 122-129, 2009.
[4] E. Hanson, L. A. Kalish, E. Bunce et al., "Use of complementary and alternative medicine among children diagnosed with autism spectrum disorder," Journal of Autism and Developmental Disorders, vol. 37, no. 4, pp. 628-636, 2007.

[5] A. Lim, N. Cranswick, S. Skull, and M. South, "Survey of complementary and alternative medicine use at a tertiary children's hospital," Journal of Paediatrics and Child Health, vol. 41, no. 8, pp. 424-427, 2005.

[6] K. M. Kelly, J. S. Jacobson, D. D. Kennedy, S. M. Braudt, M. Mallick, and M. A. Weiner, "Use of unconventional therapies by children with cancer at an urban medical center," Journal of Pediatric Hematology/Oncology, vol. 22, no. 5, pp. 412-416, 2000.

[7] J. Post-White, M. Fitzgerald, S. Hageness, and S. F. Sencer, "Complementary and alternative medicine use in children with cancer and general and specialty pediatrics," Journal of Pediatric Oncology Nursing, vol. 26, no. 1, pp. 7-15, 2009.

[8] M. Reznik, P. O. Ozuah, K. Franco, R. Cohen, and F. Motlow, "Use of complementary therapy by adolescents with asthma," Archives of Pediatrics and Adolescent Medicine, vol. 156, no. 10, pp. 1042-1044, 2002.

[9] M. J. Carlson and G. Krahn, "Use of complementary and alternative medicine practitioners by people with physical disabilities: estimates from a National US Survey," Disability and Rehabilitation, vol. 28, no. 8, pp. 505-513, 2006.

[10] K. A. Brown and D. R. Patel, "Complementary and alternative medicine in developmental disabilities," Indian Journal of Pediatrics, vol. 72, no. 11, pp. 949-952, 2005.

[11] S. L. Hyman and S. E. Levy, "Introduction: novel therapies in developmental disabilities-hope, reason, and evidence," Mental Retardation and Developmental Disabilities Research Reviews, vol. 11, no. 2, pp. 107-109, 2005.

[12] Y.-I. Shin, C.-Y. Yang, M.-C. Joo, S.-G. Lee, J.-H. Kim, and M. S. Lee, "Patterns of using complementary and alternative medicine by stroke patients at two university hospitals in Korea," Evidence-Based Complementary and Alternative Medicine, vol. 5, no. 2, pp. 231-235, 2008.

[13] M.-S. Lee, M. S. Lee, C.-Y. Yang et al., "Use of complementary and alternative medicine by rheumatoid arthritis patients in Korea," Clinical Rheumatology, vol. 27, no. 1, pp. 29-33, 2008.

[14] D. Z. Samdup, R. G. Smith, and S. Il Song, "The use of complementary and alternative medicine in children with chronic medical conditions," American Journal of Physical Medicine and Rehabilitation, vol. 85, no. 10, pp. 842-846, 2006.

[15] A. Furnham and B. Kirkcaldy, "The health beliefs and behaviours of orthodox and complementary medicine clients," British Journal of Clinical Psychology, vol. 35, no. 1, pp. 49-61, 1996.

[16] M.-S. Lee, M. S. Lee, H.-J. Lim, and S.-R. Moon, "Survey of the use of complementary and alternative medicine among Korean diabetes mellitus patients," Pharmacoepidemiology and Drug Safety, vol. 13, no. 3, pp. 167-171, 2004.

[17] C. Vincent and A. Furnham, "Why do patients turn to complementary medicine? An empirical study," British Journal of Clinical Psychology, vol. 35, no. 1, pp. 37-48, 1996.

[18] S. Gottschling, B. Gronwald, S. Schmitt et al., "Use of complementary and alternative medicine in healthy children and children with chronic medical conditions in Germany," Complementary Therapies in Medicine, vol. 21, supplement 1, pp. S61S69, 2013. 


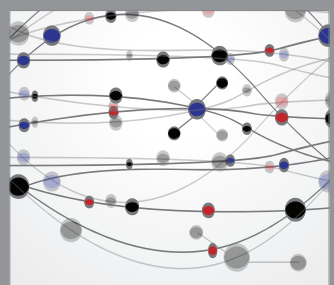

The Scientific World Journal
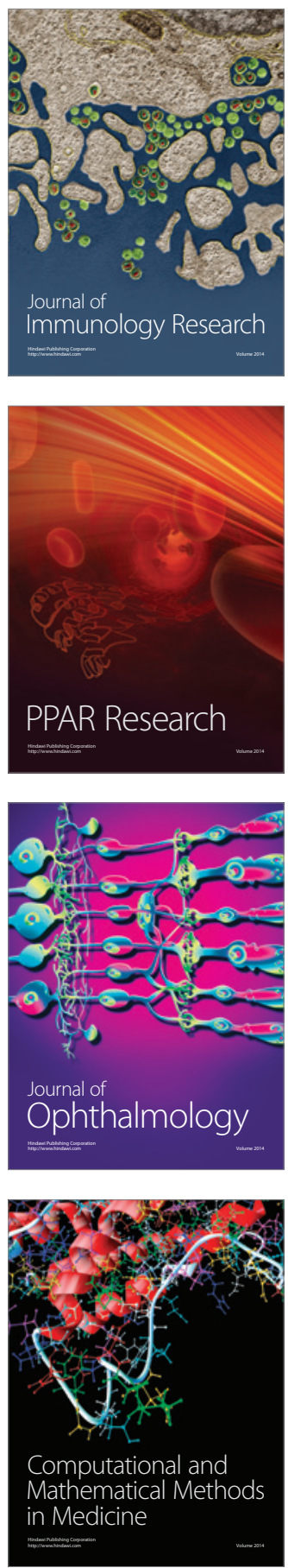

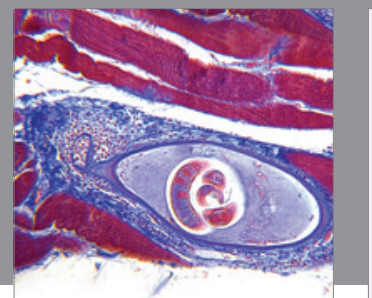

Gastroenterology

Research and Practice
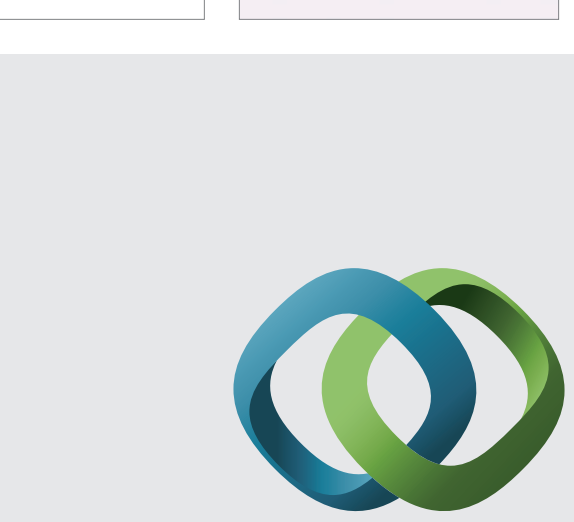

\section{Hindawi}

Submit your manuscripts at

http://www.hindawi.com
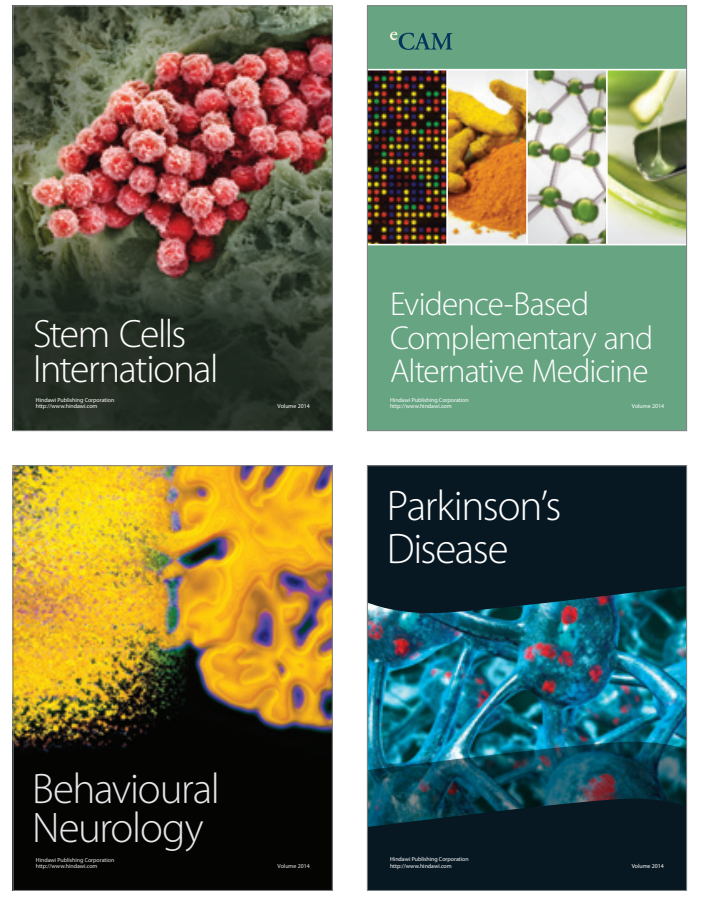
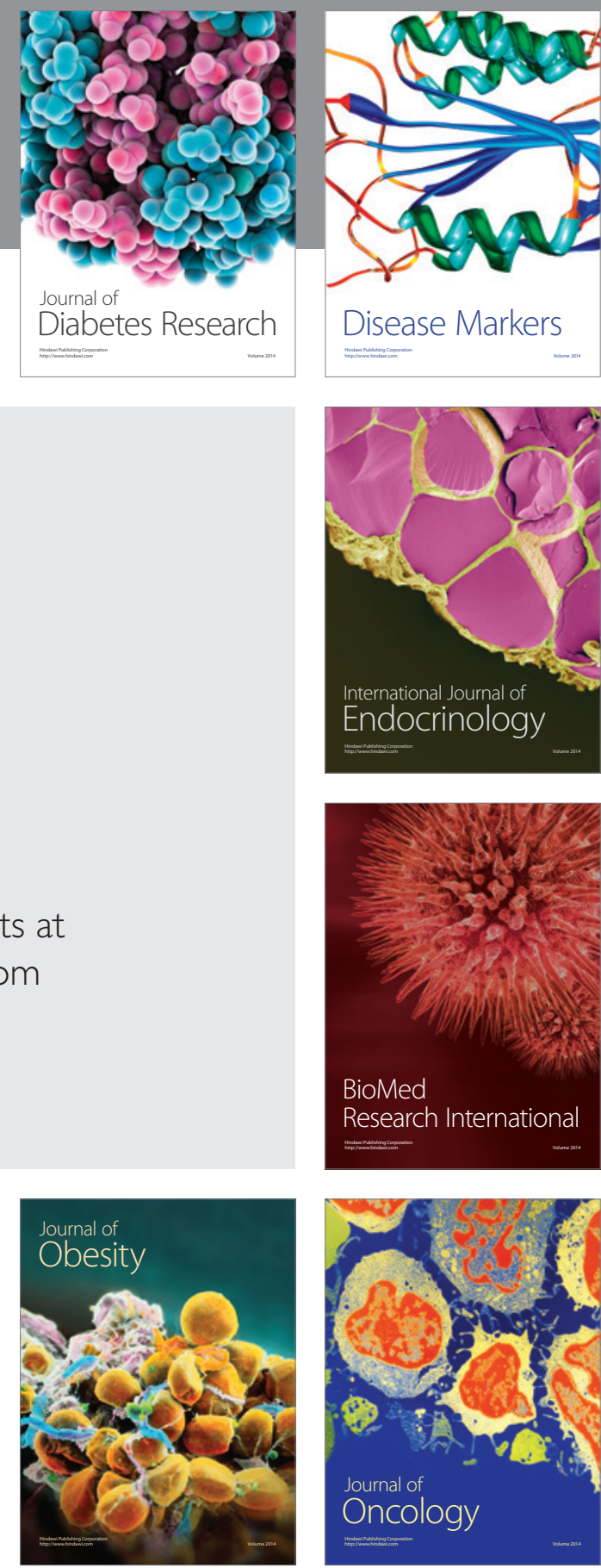

Disease Markers
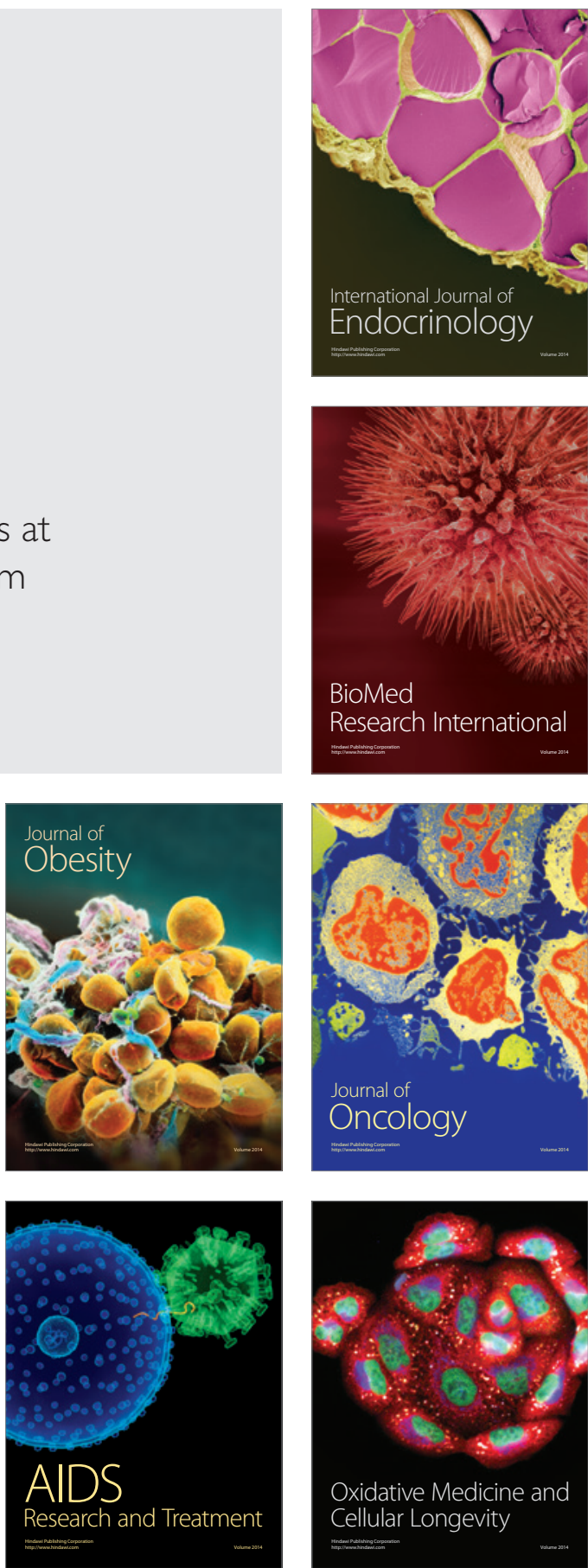